\title{
Autoestima, insatisfação corporal e internalização do ideal de magreza influenciam os comportamentos de risco para transtornos alimentares?
}

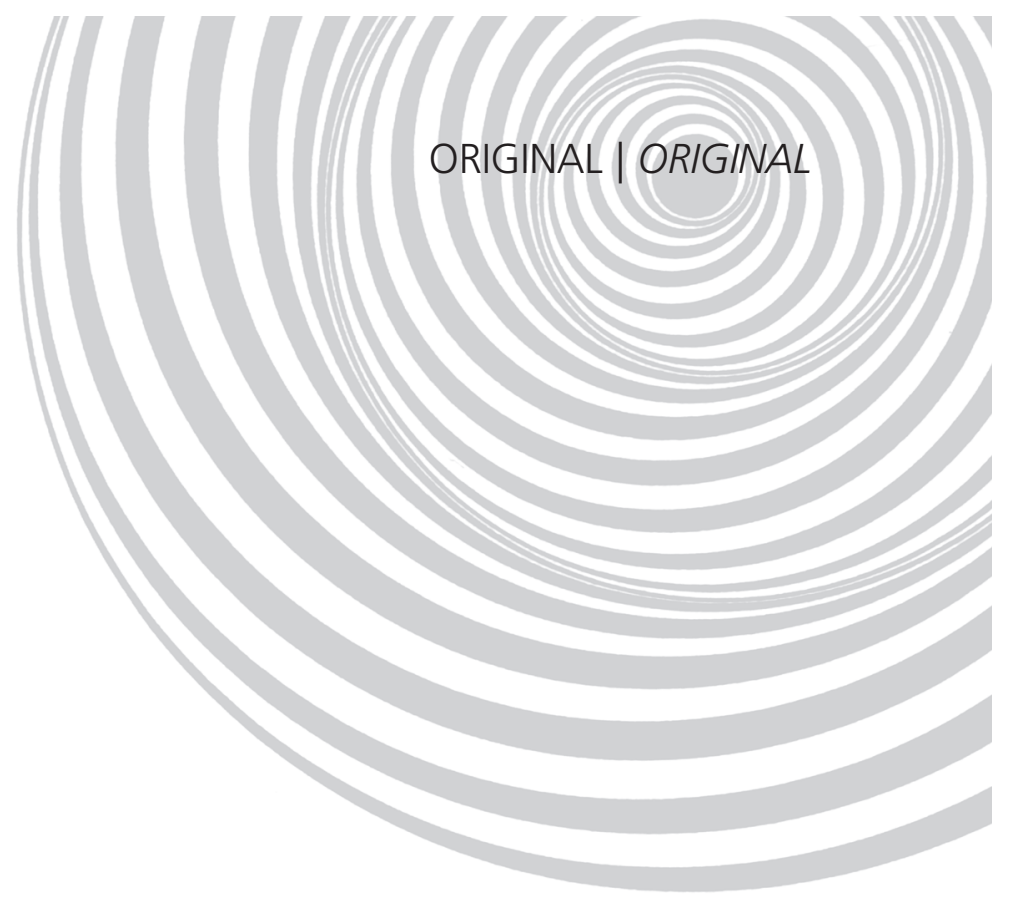

\author{
Can self-esteem, body dissatisfaction, \\ and internalization of the thinness \\ ideal influence risk behaviors \\ for eating disorders?
}

Leonardo de Sousa FORTES 1

Juliana Fernandes Filgueiras MEIRELES²

Clara Mockdece NEVES²

Sebastião Sousa ALMEIDA ${ }^{3}$

Maria Elisa Caputo FERREIRA²

RE S U M O

\section{Objetivo}

O objetivo do presente estudo foi verificar a influência da autoestima, da insatisfação corporal e da internalização do ideal de magreza nos comportamentos de risco para transtornos alimentares de adolescentes do sexo feminino.

\section{Métodos}

Participaram do estudo 471 jovens. Utilizou-se a Escala de Autoestima de Rosemberg, o Body Shape Questionnaire e o Sociocultural Attitudes Towards Appearance Questionnaire-3 para avaliar autoestima, insatisfação corporal e internalização do ideal de magreza, respectivamente. As subescalas do Eating Attitudes Test foram utilizadas para avaliar os comportamentos de risco para transtornos alimentares.

\section{Resultados}

Os resultados indicaram influência dos escores do Body Shape Questionnaire $(p<0,05)$ e da Sociocultural Attitudes Towards Appearance Questionnaire-3 $(p<0,05)$ em todas as subescalas do Eating Attitudes Test. Em contrapartida,

${ }^{1}$ Universidade Federal do Pernambuco, Núcleo de Educação Física e Ciências do Esporte, Centro Acadêmico de Vitória. R. Clóvis Beviláqua, 163/1003, Madalena, 50710-330, Recife, PE, Brasil. Correspondência para/Correspondence to: LS FORTES. E-mail: <leodesousafortes@hotmail.com>.

2 Universidade Federal de Juiz de Fora, Faculdade de Educação Física e Desportos, Departamento de Fundamentos da Educação Física. Juiz de Fora, MG, Brasil.

${ }^{3}$ Universidade de São Paulo, Faculdade de Filosofia, Ciências e Letras, Departamento de Psicologia. Ribeirão Preto, SP, Brasil. 
os achados não demonstraram influência da Escala de Autoestima de Rosemberg nos escores das subescalas do Eating Attitudes Test ( $p>0,05)$.

\section{Conclusão}

Concluiu-se que a insatisfação corporal e a internalização do ideal de magreza influenciam os comportamentos de risco para transtornos alimentares em adolescentes do sexo feminino, fato que não foi evidenciado para a autoestima.

Palavras-chave: Adolescentes. Imagem corporal. Transtornos alimentares.

\section{A B S T R A C T}

\section{Objetive}

The aim of this study was to investigate the influence of self-esteem, body dissatisfaction, and internalization of the thin ideal on disordered eating female adolescents.

\section{Methods}

Four hundred and seventy one adolescents participated in this research. The Rosenberg Self-Esteem Scale, the Body Shape Questionnaire, and the Sociocultural Attitudes Towards Appearance Questionnaire-3 were used to assess self-esteem, body dissatisfaction, and internalization of the thin ideal, respectively. The Eating Attitudes Test subscales were used to evaluate eating disorder risk behavior.

\section{Results}

The results indicated influence of the Body Shape Questionnaire $(p<0.05)$ and Sociocultural Attitudes Towards Appearance Questionnaire-3 scores $(p<0.05)$ on all subscales of the Eating Attitudes Test. In contrast, the findings did not show any significant influence of the Rosenberg Self-Esteem Scale on the Eating Attitudes Test subscales scores ( $p>0.05)$.

\section{Conclusion}

It was concluded that body dissatisfaction and internalization of the thin ideal influence eating disorder risk behavior in female adolescents; the same behavior was not observed for self-esteem.

Keywords: Adolescents. Body image. Eating disorders.

\section{N T R O D U ÇÃ O}

A adolescência é a fase que marca a transição entre a infância e a idade adulta': é uma etapa da vida do ser humano na qual se passa por um turbilhão de alterações tanto em sua forma e composição corporal, quanto em suas características psíquicas ${ }^{2,3}$. Segundo Helfert \& Warschburger ${ }^{4}$, é nesse período - entre 10 e 19 anos $^{1}$-, em que o adolescente estrutura de forma mais significativa sua imagem corporal. Esse construto pode ser entendido como a representação mental que o indivíduo tem do seu próprio corpo 5 , e um de seus componentes atitudinais é a insatisfação corporal. Esta, por sua vez, refere-se à avaliação negativa do peso e da forma física ${ }^{3}$.

De acordo com o Diagnostic and Statistical Manual of Mental Disorders - IV (DSM-IV, Manual
Diagnóstico e Estatístico de Transtornos Mentais) ${ }^{6}$, a insatisfação corporal pode ser considerada um dos fatores diagnósticos dos Transtornos Alimentares (TA), tais como anorexia e bulimia nervosa. Dessa forma, comportamento de risco para TA e insatisfação corporal estão intimamente relaciona$\operatorname{dos}^{7-8}$. Atualmente, os estudos têm verificado alta prevalência de comportamento de risco para TA em adolescentes do sexo feminino ${ }^{7,9-11}$, em decorrência do aumento dos índices de insatisfação corporal nessas jovens ${ }^{12,13}$. Os autores justificam esses índices devido à forte tendência cultural em considerar a magreza como ideal corporal feminino ${ }^{5,14}$.

Além da insatisfação corporal, os comportamentos de risco para TA são provenientes do medo de engordar e do desejo constante de perder peso ${ }^{15}$. Pais, amigos e mídia reforçam esse padrão e destacam a aceitação social como um 
benefício derivado da magreza ${ }^{4}$. A internalização do ideal sociocultural de corpo pode mediar a insatisfação corporal, que, por sua vez, influencia a adoção de comportamentos de risco para TA nas adolescentes do sexo feminino $0^{5,14}$, pois o corpo ideal destacado é, na maioria das vezes, inatingível ${ }^{13}$.

Outros aspectos psicológicos podem estar diretamente associados aos comportamentos de risco para $\mathrm{TA}^{10}$ : a autoestima tem sido investigada como um desses fatores ${ }^{16-18}$, uma vez que ela é entendida como a avaliação do indivíduo sobre o seu próprio valor, competência e adequação, e se reflete em uma atitude positiva ou negativa, em algum grau, em relação a si mesmo ${ }^{19}$. Flament et al. ${ }^{16}$ demostraram que a variabilidade da autoestima se encontra estritamente relacionada com a insatisfação corporal. Entretanto, esses autores apontaram a necessidade de mais estudos que relacionem essas variáveis ao comportamento de risco para TA.

Alves et al. ${ }^{7}$ e Scherer et al. ${ }^{8}$ argumentam que, nos últimos anos, houve um aumento dos estudos sobre sintomas e comportamentos de risco para TA, principalmente em adolescentes do sexo feminino e com ocorrência em idades cada vez mais precoces, tornando-se um sério problema de saúde pública. No entanto, ainda não são encontrados na literatura nacional estudos que investiguem a influência da autoestima, da insatisfação corporal e da internalização do ideal de magreza nos comportamentos de risco para TA de adolescentes do sexo feminino. Sbicigo et al. ${ }^{19}$ ainda acrescentam que são necessárias investigações que relacionem diferentes instrumentos de aferição psicológica a fim de contribuir para uma discussão ampliada sobre o tema.

Dessa forma, o objetivo deste estudo foi verificar a influência da autoestima, da insatisfação corporal e da internalização do ideal de magreza nos comportamentos de risco para TA de adolescentes do sexo feminino da cidade de Juiz de Fora, Minas Gerais.

\section{M É T O D O S}

Trata-se de estudo transversal, de base escolar, realizado no ano de 2012 na cidade de Juiz de Fora (MG), com adolescentes do sexo feminino na faixa etária entre 12 e 16 anos.

Segundo informações da Secretaria de Educação de Juiz de Fora, a população de adolescentes do sexo feminino com idade entre 12 e 16 anos, matriculada nas escolas dessa cidade em 2012, era de aproximadamente 35 mil jovens. Para o cálculo amostral, adotou-se prevalência de comportamentos de risco para TA de $30 \%^{8}, 5 \%$ de erro amostral e $95 \%$ de confiança, chegando-se ao tamanho amostral de 311 adolescentes. Em seguida, utilizou-se efeito de desenho igual a 1,2 para corrigir as estimativas dos modelos estatísticos. Logo, o tamanho amostral necessário foi de 373 adolescentes.

A amostra proporcional foi estratificada segundo a inserção das escolas nas regiões sociogeográficas do município (Norte, Sul, Centro) e o tipo de vinculação administrativa (pública e privada).

A seleção ocorreu aleatoriamente, por meio de sorteio simples. Realizou-se o sorteio das escolas em cada região em razão do tipo de vinculação administrativa. As escolas foram selecionadas valendo-se da relação fornecida pelo setor de estatística da Secretaria de Educação de Minas Gerais (MG). A amostra final da pesquisa foi distribuída em 12 pontos diferentes de coleta (6 escolas privadas e 6 públicas) e constituída por adolescentes do sexo feminino presentes nas escolas nos dias da coleta e cujos pais autorizaram a participação na pesquisa. Foram incluídas na pesquisa somente as jovens que apresentaram o Termo de Consentimento Livre e Esclarecido (TCLE) assinado pelo responsável e que estavam regularmente matriculadas no ensino fundamental/médio na cidade de Juiz de Fora (MG) no ano de 2012.

Participaram do estudo 489 jovens, sendo 18 destas excluídas por não responderem aos questionários por completo, o que totalizou uma amostra final de 471 adolescentes. 
A variável autoestima foi avaliada por intermédio da Escala de Autoestima de Rosenberg $(E A R)^{19}$, composta por 10 itens, com três opções de respostas do tipo Likert $(1=$ concordo fortemente, 2 = concordo/discordo, 3 = discordo fortemente). $\mathrm{O}$ instrumento apresenta dois fatores: $\mathrm{o}$ fator 1 agrupa seis itens relacionados à autoestima positiva, e o fator 2, quatro itens referentes à autoestima negativa. Com relação à pontuação, quanto maior o escore obtido na escala, maior o nível de autoestima do indivíduo. Em virtude da análise estatística, utilizou-se a mediana da EAR para classificar as adolescentes com alta e baixa autoestima. Assim, as meninas com escore maior ou igual a 11 foram inseridas no grupo "alta autoestima" (EAR+). A versão da escala utilizada foi validada para adolescentes brasileiros, e sua análise de consistência interna revelou um alpha de 0,70. Para a presente amostra, a consistência interna foi avaliada pelo alpha de Cronbach, obtendo-se valor equivalente a 0,71.

Para avaliar a insatisfação e as preocupações com a forma do corpo, foi utilizado o Body Shape Questionnaire (BSQ) validado para a população de adolescentes brasileiros por Conti et al. ${ }^{3}$. O questionário é composto por 34 questões, em escala tipo Likert: a avaliada aponta com que frequência, nas últimas quatro semanas, vivenciou os eventos propostos pelas alternativas. As respostas variam de 1 (nunca) a 6 (sempre), sendo a soma das pontuações de cada item o escore final da escala. A classificação dos resultados do BSQ é dividida em quatro níveis de insatisfação corporal. A pontuação abaixo de 80 indica ausência de insatisfação; entre 80 e 110, insatisfação leve; entre 111 e 140, insatisfação moderada, e acima de 140 indica grave insatisfação corporal. Para as análises estatísticas, agruparam-se as classificações de insatisfação "leve", "moderada" e "grave" como "insatisfeitas". Para a amostra da presente pesquisa, identificou-se valor de consistência interna de 0,96 para o BSQ, obtido por meio do alpha de Cronbach.

A fim de se investigar a internalização do ideal de magreza e por quais mediadores o ambiente sociocultural exerce sua influência sobre a imagem corporal, foi utilizado o Sociocultural Attitudes Towards Appearance Questionnaire-3 (SATAQ-3) ${ }^{14}$. O questionário é composto por 30 perguntas em escala do tipo Likert com 5 opções de resposta ("Discordo totalmente" até "Concordo totalmente"). O instrumento apresenta 4 subescalas referentes à: internalização do ideal de corpo atlético; internalização geral dos padrões socialmente estabelecidos; pressão exercida por esses padrões; mídia como fonte de informações sobre a aparência. O escore total do questionário é calculado pela soma das respostas, considerando que todas as questões que apresentam a palavra "não" em seu enunciado têm pontuações invertidas. Quanto maior a pontuação obtida, maior a influência dos aspectos socioculturais na imagem corporal. Em razão da análise estatística, utilizou-se a mediana do SATAQ-3 para classificar as adolescentes com alta e baixa internalização do ideal de magreza. Logo, as meninas com escore maior ou igual a 69 foram inseridas no grupo "alta internalização do ideal de magreza" (SATAQ+). A análise da consistência interna do questionário no estudo de validação para adultos jovens foi de 0,91. Para a amostra do presente estudo, o teste de alpha de Cronbach foi igual a 0,84.

Para a identificação da existência de comportamento de risco para TA, foi aplicado o Eating Attitudes Test (EAT-26), validado para meninas adolescentes por Bighetti et al. ${ }^{9}$. O questionário é composto por 26 questões distribuídas em três fatores: 1) dieta: diz respeito à recusa patológica a alimentos com alto teor calórico e preocupação com a aparência física; 2) bulimia e preocupação com os alimentos: referem-se a episódios de compulsão alimentar, seguidos por comportamentos purgativos para perda/controle de peso corporal e, 3) autocontrole oral: reflete o autocontrole em relação à comida e avalia as forças ambientais e sociais estimulantes à ingestão alimentar. A avaliada tem, em cada item, seis opções de resposta que variam de 0 (poucas vezes, quase nunca e nunca) a 3 (sempre). A única questão que apresenta pontuação em ordem reversa é a 25 . A pontuação do EAT-26 é feita pela soma de seus itens. Quando o total de pontos obtidos é maior 
ou igual a 21, a presença de transtornos de conduta alimentar é considerada positiva. No estudo de validação, Bighetti et al. ${ }^{9}$ evidenciaram consistência interna de 0,82. Para a presente amostra, encontrou-se valor para a consistência interna de 0,87, avaliada pelo alpha de Cronbach.

Os diretores de 14 escolas (7 privadas e 7 públicas) foram convidados a participar da pesquisa, sendo informados sobre objetivos e procedimentos do estudo. No entanto, somente 12 destes ( 6 de escolas privadas e 6 de públicas) concordaram em liberar as alunas para participação nas coletas. Após a autorização da direção das escolas, foram realizadas reuniões com cada uma das turmas para explicar os objetivos e procedimentos necessários para inclusão das escolares no estudo. Foi entregue o TCLE às adolescentes, pedindo-lhes que o devolvessem devidamente assinado pelos responsáveis na semana seguinte, em caso de assentimento de sua participação voluntária.

A pesquisa foi realizada em um único momento. As alunas responderam aos instrumentos (EAR, BSQ, SATAQ-3 e EAT-26), etapa realizada em grupo, por um único pesquisador, que padronizou as explicações verbais.

Este estudo obteve aprovação do Comitê de Ética e Pesquisa em Seres Humanos da Faculdade de Filosofia, Ciências e Letras de Ribeirão Preto, Universidade de São Paulo (Protocolo $n^{\circ} 109.971$ ) - de acordo com a Lei n 196/96 do Conselho Nacional de Saúde.

\section{Análise estatística}

O teste Kolmogorov Smirnov foi utilizado para analisar a distribuição dos escores do EAT-26. Em razão da não violação paramétrica, foram utilizadas medidas de tendência central (média e mediana), dispersão (mínimo, máximo e desvio-padrão) e frequência (absoluta e relativa) para descrever as variáveis da investigação. Conduziu-se a regressão linear múltipla stepwise em três blocos, com o controle da idade para averiguar a influência das variáveis independentes
(EAR, BSQ e SATAQ-3) sobre os escores das subescalas do EAT-26. Ademais, a Multivariate Analysis of Variance (Manova, Análise Multivariada de Covariância) foi utilizada para comparar os escores das subescalas do EAT-26 em razão das classificações de autoestima (EAR $\geq 11=$ alta autoestima e EAR $<11$ = baixa autoestima), insatisfação corporal (BSQ $\geq 80$ = insatisfeita e BSQ $<80$ = satisfeita) e internalização do ideal de magreza (SATAQ-3 $\geq 69$ = alta internalização do ideal de magreza e SATAQ-3 $<69$ = baixa internalização do ideal de magreza), inserindo-se a idade como covariável. O post hoc de Bonferroni foi conduzido para identificar possíveis diferenças. Todos os dados foram analisados no software Statistical Package for the Social Sciences (SPSS) 20.0, e adotou-se significância de 5\%.

\section{RES U LTA DOS}

Os valores descritivos (mínimo, máximo, média e desvio-padrão) de todas as variáveis da investigação podem ser visualizados na Tabela 1. No que tange à EAR, os achados da presente investigação apontaram que 62,3\% das adolescentes demonstraram baixa autoestima. Em relação ao $\mathrm{BSQ}$, os resultados do estudo evidenciaram que 23,2\% das adolescentes manifestaram insatisfação corporal (Tabela 1). A respeito do SATAQ-3, 46,4\% das avaliadas demonstraram alta internalização do ideal de magreza. Em adição, os achados concernentes ao EAT-26 indicaram prevalência de 21,7\% para os comportamentos de risco para TA.

O modelo de regressão evidenciou influência do BSQ $\left(F_{(1,470)}=121,33 ; p=0,001\right)$ e do SATAQ-3 $\left(F_{(1,470)}=15,23 ; p=0,001\right)$ na subescala Dieta do EAT-26 (Tabela 2). Assim, esses resultados indicaram que a insatisfação corporal e a internalização do ideal sociocultural de magreza explicaram 64 e 19\%, respectivamente, da variância da restrição alimentar em adolescentes do sexo feminino. No entanto, a EAR não apontou influência estatisticamente significativa na subescala Dieta $\left(F_{(1,470)}=0,47 ; p=0,49\right)$. Logo, esses achados demonstraram que a autoestima não impactou 
Tabela 1. Resultados descritivos dos escores das escalas utilizadas e idade de adolescentes do sexo feminino ( $n=471$ ). Juiz de Fora (MG), 2013.

\begin{tabular}{lcccc}
\hline Variável & Mínimo & Máximo & Média & DP \\
\hline EAR & 0 & 16 & 10,28 & 2,94 \\
BSQ & 34 & 186 & 65,26 & 33,36 \\
SATAQ-3 & 30 & 123 & 68,87 & 20,52 \\
EAT-26 & 0 & 54 & 13,33 & 11,68 \\
Idade (anos) & 12 & 16 & 13,03 & 1,59 \\
\hline
\end{tabular}

Nota: DP: Desvio-Padrão; EAR: Escala de Autoestima de Rosemberg; BSQ: Body Shape Questionnaire; SATAQ-3: Sociocultural Attitudes Towards Appearance Questionnaire-3; EAT-26: Eating Attitudes Test.

Tabela 2. Regressão linear múltipla utilizando a EAR, o BSQ e a SATAQ-3 como variáveis explicativas sobre a variância da subescala Dieta do EAT-26 em adolescentes do sexo feminino ( $n=471$ ). Juiz de Fora (MG), 2013.

\begin{tabular}{lcccccc}
\hline Variável & Bloco & $B$ & $R$ & $R^{2}$ & $R^{2^{*}}$ & $p$-valor \\
\hline EAR & 1 & 0,22 & 0,08 & 0,007 & 0,008 & $\leq 0,49$ \\
BSQ & 2 & 0,18 & 0,80 & 0,64 & 0,64 & $\leq 0,01$ \\
SATAQ-3 & 3 & 0,16 & 0,43 & 0,19 & 0,17 & $\leq, 01$ \\
Todos & & 3,30 & 0,80 & 0,65 & 0,64 & $\leq 0,01$ \\
\hline
\end{tabular}

Nota: $R^{2^{*}}: R^{2}$ ajustado; B: Beta; $R$ : $R$ de Pearson; EAR: Escala de Autoestima de Rosemberg; BSQ: Body Shape Questionnaire; SATAQ-3: Sociocultural Attitudes Towards Appearance Questionnaire-3; EAT-26: Eating Attitudes Test.

Tabela 3. Regressão linear múltipla utilizando a EAR, o BSQ e a SATAQ-3 como variáveis explicativas sobre a variância da subescala Bulimia e Preocupação com Alimentos do EAT-26 em adolescentes do sexo feminino ( $n=471)$. Juiz de Fora (MG), 2013.

\begin{tabular}{lcccccc}
\hline Variável & Bloco & $B$ & $R$ & $R^{2}$ & $R^{2^{*}}$ & $p$-valor \\
\hline EAR & 1 & 0,03 & 0,4 & 0,02 & 0,01 & $\leq 0,74$ \\
BSQ & 2 & 0,12 & 0,64 & 0,41 & 0,40 & $\leq 0,01$ \\
SATAQ-3 & 3 & 0,09 & 0,29 & 0,09 & 0,07 & $\leq, 02$ \\
Todos & & 3,42 & 0,65 & 0,43 & 0,40 & $\leq 0,01$ \\
\hline
\end{tabular}

Nota: $R^{2^{*}}: R^{2}$ ajustado; B: Beta; $R$ : $R$ de Pearson; EAR: Escala de Autoestima de Rosemberg; BSQ: Body Shape Questionnaire; SATAQ-3: Sociocultural Attitudes Towards Appearance Questionnaire-3; EAT-26: Eating Attitudes Test.

sobre a restrição alimentar das escolares da presente amostra.

A Tabela 3 ilustra o modelo de regressão que utilizou a subescala Bulimia e Preocupação com Alimentos como variável critério. Os resultados apontaram influência estatisticamente significativa do BSQ $\left(F_{(1,470)}=46,19 ; p=0,001\right)$ e do SATAQ-3 $\left(F_{(1,470)}=6,35 ; p=0,014\right)$. Logo, os achados demonstraram que a insatisfação corporal $(41 \%)$ e a internalização do ideal sociocultural de magreza (9\%) influenciaram a compulsão alimentar e os comportamentos purgativos de adolescentes do sexo feminino. Ressalta-se que a $\operatorname{EAR}\left(F_{(1,470)}=0,11 ; p=0,74\right)$ não explicou a variância da subescala Bulimia e Preocupação com Alimentos. Todavia, todas as variáveis independentes juntas (EAR, BSQ e SATAQ-3) impactaram sobre $43 \%\left(F_{(1,470)}=16,20 ; p=0,001\right)$ da variância da subescala Bulimia e Preocupação com Alimentos do EAT-26.

O último modelo de regressão, que utilizou a subescala Autocontrole Oral como variável dependente, pode ser observado na Tabela 4. O BSQ $\left(F_{(1,470)}=7,81 ; p=0,007\right)$ e a SATAQ-3 
$\left(F_{(1,470)}=8,78 ; p=0,004\right)$ demonstraram impacto significante sobre os escores da subescala Autocontrole Oral. Nesse sentido, a $\operatorname{EAR}\left(F_{(1,470)}=0,31\right.$; $p=0,57)$ não explicou a variância do autocontrole em relação à comida em adolescentes do sexo feminino. Contudo, salienta-se que o modelo de regressão com todas as variáveis explicativas (EAR, BSQ e SATA-3) inseridas em um único bloco $\left(F_{(1,470)}=3,87 ; p=0,0013\right)$ modulou $15 \%$ da variância da subescala Autocontrole Oral do EAT-26.

No que concerne às comparações das subescalas do EAT-26 em razão dos grupos de autoestima, os resultados indicaram semelhanças na frequência de restrição alimentar $\left(F_{(1,470)}=1,14\right.$; $p=0,69)$ e de influência ambiental na ingestão alimentar $\left(F_{(1,470)}=1,99 ; p=38\right)$ em adolescentes com alta e baixa autoestima (Tabela 5). No entanto, as jovens com baixa autoestima demonstraram maior frequência de comportamento purgativo quando comparadas às adolescentes com alta autoestima $\left(F_{(1,470)}=5,66 ; p=0,05\right)$. A respeito da insatisfação corporal, a Manova apontou alguns achados que merecem destaque (Tabela 5): 1) evidenciou-se diferença de escore nas subescalas Dieta $\left(F_{(1,470)}=158,02 ; p=0,001\right)$ e Bulimia e Preocupação com Alimentos $\left(F_{(1,470)}=63,91\right.$; $p=0,001$ ) entre as adolescentes satisfeitas e insa-

Tabela 4. Regressão linear múltipla utilizando a EAR, o BSQ e a SATAQ-3 como variáveis explicativas sobre a variância da subescala Autocontrole Oral do EAT-26 em adolescentes do sexo feminino ( $n=471)$. Juiz de Fora (MG), 2013.

\begin{tabular}{lcccccc}
\hline Variável & Bloco & $B$ & $R$ & $R^{2}$ & $R^{2^{*}}$ & $p$-valor \\
\hline EAR & 1 & 0,09 & 0,07 & 0,005 & 0,004 & $\leq 0,57$ \\
BSQ & 2 & 0,10 & 0,32 & 0,10 & 0,09 & $\leq 0,01$ \\
SATAQ-3 & 3 & 0,08 & 0,34 & 0,12 & 0,11 & $\leq 0,01$ \\
Todos & & 4,44 & 0,39 & 0,15 & 0,12 & $\leq 0,01$ \\
\hline
\end{tabular}

Nota: $R^{2^{*}}: R^{2}$ ajustado; B: Beta; $R$ : $R$ de Pearson; EAR: Escala de Autoestima de Rosemberg; BSQ: Body Shape Questionnaire; SATAQ-3: Sociocultural Attitudes Towards Appearance Questionnaire-3; EAT-26: Eating Attitudes Test.

Tabela 5. Comparação dos escores das subescalas do EAT-26 em função dos grupos de autoestima, insatisfação corporal e internalização do ideal de magreza em adolescentes do sexo feminino ( $n=471)$. Juiz de Fora (MG), 2013.

\begin{tabular}{|c|c|c|c|c|c|}
\hline \multirow{3}{*}{$\frac{\text { Variável }}{\text { Autoestima }}$} & \multicolumn{4}{|c|}{ Grupo } & \multirow[t]{3}{*}{$p$-valor } \\
\hline & \multicolumn{2}{|c|}{ Alta $(E A R \geq 11)$} & \multicolumn{2}{|c|}{ Baixa $(E A R<11)$} & \\
\hline & M & $\mathrm{DP}$ & M & DP & \\
\hline Dieta & 7,45 & 1,51 & 8,37 & 1,17 & $\leq 0,69$ \\
\hline Bul e PA & 1,02 & 0,51 & 2,24 & 0,39 & $\leq 0,05$ \\
\hline AC Oral & 2,97 & 0,78 & 3,85 & 0,60 & $\leq 0,38$ \\
\hline \multirow[t]{2}{*}{ Insatisfação } & \multicolumn{2}{|c|}{ Satisfeita (BSQ <80) } & \multicolumn{2}{|c|}{ Insatisfeita (BSQ $\geq 80$ ) } & \\
\hline & M & $\mathrm{DP}$ & M & $\mathrm{DP}$ & \\
\hline Dieta & 5,53 & 0,85 & 16,28 & 1,56 & $\leq 0,01$ \\
\hline & 0,95 & 0,30 & 4,53 & 0,54 & $\leq 0,01$ \\
\hline AC Oral & 3,20 & 0,54 & 4,55 & 0,99 & $\leq 0,24$ \\
\hline \multirow[t]{2}{*}{ Internalização } & \multicolumn{2}{|c|}{ Baixa (SATAQ-3 <69) } & \multicolumn{2}{|c|}{ Alta (SATAQ-3 $\geq 69)$} & \\
\hline & M & $\mathrm{DP}$ & M & DP & \\
\hline Dieta & 5,77 & 1,19 & 10,63 & 1,28 & $\leq 0,01$ \\
\hline Bul e PA & 1,20 & 0,42 & 2,44 & 0,45 & $\leq 0,04$ \\
\hline AC Oral & 2,77 & 0,64 & 4,38 & 0,69 & $\leq 0,05$ \\
\hline
\end{tabular}

Nota: Bul e PA: Bulimia e Preocupação com Alimentos; AC: Autocontrole; M: Média; DP: Desvio-Padrão; EAR: Escala de Autoestima de Rosemberg; BSQ: Body Shape Questionnaire; SATAQ-3: Sociocultural Attitudes Towards Appearance Questionnaire-3. 
tisfeitas com o corpo, e 2) as jovens satisfeitas e insatisfeitas com o corpo demonstraram semelhanças nos escores da subescala Autocontrole Oral $\left(F_{(1,470)}=1,38 ; p=0,24\right)$. Por fim, em relação à internalização do ideal de magreza, os achados da Manova indicaram diferenças para as subescalas Dieta $\left(F_{(1,470)}=95,73 ; p=0,001\right)$, Bulimia e Preocupação com Alimentos $\left(F_{(1,470)}=32,89\right.$; $p=0,04)$ e Autocontrole Oral $\left(F\left(_{(1,470)}=19,22\right.\right.$; $p=0,05)$ entre adolescentes com alta e baixa internalização do ideal de magreza (Tabela 5).

\section{I S C U S S Ã O}

A presente pesquisa teve como objetivo verificar a influência da autoestima, da insatisfação corporal e da internalização do ideal de magreza nos comportamentos de risco para TA de adolescentes do sexo feminino da cidade de Juiz de Fora, Minas Gerais. Vale destacar a importância da avaliação de possíveis fatores que possam influenciar os comportamentos alimentares inadequados, o que amplia os conhecimentos sobre o tema com o propósito de incentivar ações preventivas que promovam comportamentos saudáveis, especialmente nas jovens. Entretanto, por meio de busca nas principais bases de dados de artigos científicos (Web of Science, Scopus, MedLine e SciELO), não foram encontradas pesquisas nacionais que avaliassem as variáveis psicológicas deste estudo e suas influências no comportamento alimentar.

Concernente à prevalência dos comportamentos de risco para TA, esse achado está dentro dos valores encontrados em pesquisas nacionais que empregaram o mesmo instrumento. Alves et al. ${ }^{7}$, Scherer et al. ${ }^{8}$ Fortes et al. ${ }^{10,20,21}$, Martins et $a l .{ }^{12}$, relataram, em amostras de adolescentes do sexo feminino, frequência de comportamentos de risco para TA de aproximadamente $20 \%$. Os pesquisadores têm justificado tais valores, pois parece que o sexo feminino tem enaltecido a aparência física direcionada para o baixo peso, o que pode ter consequências nos comportamentos de risco para $\mathrm{TA}^{11,16}$.
Estudos têm encontrado prevalência relativamente elevada de insatisfação corporal em adolescentes do sexo feminino 7,8,12,22, o que justifica o resultado identificado na presente investigação. Há evidências de que as mensagens socioculturais direcionadas para a magreza transmitidas pelos meios de comunicação possam ser responsáveis pelas preocupações acerca da imagem corporal em adolescentes do sexo feminino ${ }^{5,23}$.

Segundo a literatura da área, as adolescentes tendem adotar dietas restritivas a fim de reduzir o peso corporal para alcançar o ideal de corpo magro difundido na sociedade atual ${ }^{4,10,22,24}$. Corroborando esses dados, os achados do presente estudo demonstraram que adolescentes insatisfeitas e que tinham o ideal de magreza internalizado estavam mais relacionadas a padrões de restrição alimentar (Tabela 2).

Em contrapartida, a autoestima não modulou a subescala Dieta. É provável que a autoestima não esteja relacionada com a adoção de condutas de restrição da ingestão de alimentos com alto teor calórico em adolescentes do sexo feminino. Ressalta-se que a tentativa de relacionar a autoestima aos comportamentos de risco para TA tem sido realizada em âmbito internacional. Em adolescentes canadenses do sexo feminino, Flament et al. ${ }^{16}$ buscaram relações entre essas variáveis e encontraram que a autoestima foi responsável por uma proporção significativa da variância nos escores da subescala Dieta. Cabe salientar, no entanto, que embora a presente pesquisa seja inovadora do ponto de vista nacional, o resultado encontrado não pode ser generalizado para todas as adolescentes brasileiras.

Quanto aos fatores que modularam a subescala Bulimia, a partir do modelo de regressão, os resultados indicaram influência somente da insatisfação corporal e da internalização do ideal de magreza. Os escores do BSQ e da SATAQ-3 explicaram em 41 e 9\%, respectivamente, a variância dos comportamentos bulímicos nas adolescentes. Nesse sentido, a literatura demonstra que a insatisfação com o peso 
e a aparência física é considerada fator de risco de alta relevância para os sintomas de bulimia nervosa ${ }^{5,24}$. Acrescenta-se, ainda, que as adolescentes que costumam ter preferência por alimentos considerados de alto teor calórico podem utilizar a voracidade alimentar seguida de comportamentos purgativos em razão de se sentirem pressionadas por pais, amigos e mídia em reduzirem o peso corporal ${ }^{16,25}$.

Já a autoestima não exerceu influência nos comportamentos alimentares compulsivos e purgativos. Essa variável psicológica tem sido investigada como um dos fatores que podem estar diretamente associados ao comportamento de risco para $T A^{16-18}$. Além disso, tem sido encontrada estreita relação desta com a insatisfação corpora ${ }^{23,26}$, que, como supramencionado, é critério diagnóstico para os TA. Dessa forma, esperava-se que a autoestima modulasse comportamentos bulímicos. Segundo Johnson et al. ${ }^{27}$, a baixa autoestima pode ser um fator de risco para os distúrbios alimentares. Todavia, a ausência de relação direta entre autoestima e comportamentos compulsivos e purgativos evidenciada na presente investigação pode ter a seguinte explicação: considerando modelos teóricos da etiologia dos comportamentos alimentares ${ }^{5,16}$, a insatisfação corporal media a relação entre autoestima e comportamentos alimentares associados à bulimia. Logo, a autoestima pode não apresentar relação direta com os comportamentos compulsivos e purgativos, o que pode explicar os achados da presente pesquisa. Contudo, são sugeridos estudos que melhor esclareçam essa relação.

No que concerne ao último modelo de regressão, os resultados indicaram, mais uma vez, que apenas o BSQ e o SATAQ-3 exerceram influência sobre os escores da subescala Autocontrole Oral do EAT-26, ao contrário da EAR. Dessa forma, a partir dos dados encontrados, parece que as adolescentes que mais controlam os tipos e os alimentos que costumam ingerir são aquelas insatisfeitas e influenciadas pelo padrão culturalmente aceito.

No que concerne às comparações das subescalas do EAT-26 em razão das classificações dicotômicas da EAR, os achados apontaram semelhanças de restrição alimentar e autocontrole sobre os alimentos. Todavia, as jovens com baixa autoestima indicaram maior frequência de comportamentos compulsivos e purgativos em comparação com as adolescentes com elevada autoestima. Talvez as adolescentes sofram de compulsão alimentar, façam uso de laxantes/ diuréticos e provoquem vômitos na tentativa de reduzirem a magnitude dos sentimentos de inutilidade e fracasso. Segundo Flament et al. ${ }^{16}$, as adolescentes com baixa autoestima podem ingerir exageradamente alimentos ricos em açúcar, pois estes induzem secreção de neurotransmissores, como a serotonina, que produzem sensações de bem-estar e felicidade. No entanto, vale salientar que após a voracidade alimentar, as adolescentes poderão buscar estratégias de controle do peso corporal, como os comportamentos purgativos ${ }^{5}$.

A respeito das comparações das subescalas do EAT-26 em função das classificações do BSQ, os resultados demonstraram que as jovens insatisfeitas com o corpo adotavam com maior frequência as dietas restritivas e os comportamentos compulsivos e purgativos. De acordo com Alves et al. ${ }^{7}$ e Scherer et al. ${ }^{8}$, a insatisfação com o peso e a aparência física é fator de primeira ordem para o desencadeamento de comportamentos alimentares utilizados por pacientes com diagnóstico clínico de TA. Ressalta-se, sobretudo, que os resultados da presente investigação não indicaram diferenças de autocontrole sobre os alimentos entre jovens satisfeitas e insatisfeitas com o corpo. Assim, possivelmente o autocontrole sobre os alimentos e a percepção da pressão para o controle de ingestão alimentar não dependem dos níveis de depreciação com o peso e a aparência física em adolescentes do sexo feminino. As forças ambientais e sociais estimulantes à ingestão alimentar possivelmente estejam mais relacionadas ao estado nutricional. De acordo com Forrester-Knauss et al. ${ }^{28}$, as adolescentes com sobrepeso e/ou obesidade se sentem mais pressionadas em controlar a alimentação em comparação às com peso normal. Considerando que o estado nutricional tem associação com a insatisfação cor- 
poral $^{3,29}$, é possível que a relação do autocontrole oral sobre os alimentos e das forças ambientais para a ingestão alimentar com a insatisfação corporal seja mediada pelo estado nutricional. No entanto, pesquisas necessitam ser desenvolvidas para tornar esse tópico menos obscuro.

Em relação às comparações das subescalas do EAT-26 em razão das classificações do SATAQ-3, o presente estudo evidenciou maior frequência de restrição alimentar, compulsão alimentar e percepção de forças ambientais para a ingesta alimentar em adolescentes com elevada internalização do ideal de magreza. De fato, pesquisas têm identificado comportamentos de restrição alimentar em jovens que desejam ser magras ${ }^{4,5}$. Ademais, estudos têm apontado que as adolescentes cuja meta é ser magra como as atrizes e as modelos de passarela costumam utilizar métodos inadequados para a diminuição do peso corporal, tais como: vômitos autoinduzidos e prática extenuante de atividade física ${ }^{11,16}$. Por fim, pesquisadores sugerem que as adolescentes facilmente influenciadas pelo ambiente sociocultural (pais, amigos e mídia) geralmente sentem que estão sendo pressionadas em adotar comportamentos direcionados ao emagrecimento ${ }^{14}$, o que, de certo modo, explica os achados da presente investigação.

Algumas limitações devem ser destacadas. Inicialmente, a versão utilizada do SATAQ-3 foi validada apenas para universitários brasileiros, que não é a população-alvo deste estudo. Entretanto, a elevada consistência interna desse instrumento entre as adolescentes participantes desta investigação dão indícios de boa qualidade psicométrica da escala também nessa população. Dessa forma, é incentivada pesquisa que busque atestar a validade e a confiabilidade desse instrumento para adolescentes. Destaca-se também o uso de questionários autopreenchíveis, pois pesquisadores afirmam que os indivíduos podem não responder com fidedignidade a ferramentas autoaplicáveis ${ }^{2}$, e os resultados podem, portanto, não refletir a realidade do contexto avaliado, visto que o resultado final é fruto de respostas subjetivas. Entretanto, pesquisadores destacam a relevância desses instrumentos desde que tenham suas qualidades psicométricas asseguradas ${ }^{5,25}$.

\section{O N CLUSÃ O}

Os achados permitiram concluir que a insatisfação corporal e a internalização do ideal de magreza estiveram relacionadas às três subescalas do EAT-26 (Dieta, Bulimia e Autocontrole Oral), ao contrário da autoestima que não modulou as subescalas desse instrumento. Entretanto, as adolescentes com baixa autoestima estiveram mais propensas a comportamentos bulímicos; as insatisfeitas, a restrições alimentares e comportamentos compulsivos e purgativos; e aquelas que mais internalizaram o ideal de magreza estiveram mais vulneráveis à Dieta, Bulimia e Autocontrole Oral. A partir desses resultados, reforça-se a necessidade de mais estudos que avaliem relações de outras variáveis psicológicas com comportamento de risco para TA. Por fim, sugere-se a implementação de ações que promovam comportamentos saudáveis, como a realização de palestras educativas em escolas sobre o tema "mídia, corpo e saúde" e discussões em sala de aula com profissionais especializados das áreas de Nutrição, Psicologia e Psiquiatria.

\section{OLABORADORES}

LS FORTES elaborou o projeto de pesquisa, coletou os dados e redigiu o artigo. JFF MEIRELES e CM NEVES auxiliaram na coleta dos dados, análise dos dados e revisão do artigo. SS ALMEIDA procedeu análises estatísticas e revisou o artigo. MEC FERREIRA orientou todo o trabalho e revisou o artigo.

\section{REFERÊ NCIAS}

1. World Health Organization. Development of a WHO growth reference for school-aged children and adolescents. Bull World Health Organ. 2007; 85(9):660-7. http://dx.doi.org/10.1590/S0042-96 862007000900010 
2. Fortes LS, Almeida SS, Ferreira MEC. Processo maturacional, insatisfação corporal e comportamento alimentar inadequado em jovens atletas. Rev Nutr. 2012, 5(5):575-86. http://dx.doi.org/10.1590/S14 15-52732012000500003

3. Conti MA, Cordás TA, Latorre MRDO. Estudo de validade e confiabilidade da versão brasileira do Body Shape Questionnaire (BSQ) para adolescentes. Rev Bras Saúde Mater Infant. 2009; 9(3):331-8. http://dx.doi.org/10.1590/S1519-38292009000 300012

4. Helfert S, Warschburger P. A prospective study on the impact of peer and parental pressure on body dissatisfaction in adolescent girls and boys. Body Image. 2011; 8(1):101-9. http://dx.doi.org/10.10 16/j.bodyim.2011.01.004

5. Rodgers R, Cabrol H, Paxton SJ. An exploration of the tripartite influence model of body dissatisfaction and disordered eating among Australian and French college women. Body Image. 2011; 8(1):208-15. http://dx.doi.org/10.1016/j.bodyim.2011.04.009

6. American Psychiatry Association. Diagnostic and statistical manual of mental disorders. $4^{\text {th }}$ ed. Washington (DC): American Psychiatric Association; 1994.

7. Alves E, Vasconcelos FAG, Calvo MCM, Neves J. Prevalence of symptoms of anorexia nervosa and dissatisfaction with body image in female adolescents in Florianópolis, Santa Catarina, Brazil. Cad Saúde Pública. 2008; 24(3):503-12. http:// dx.doi.org/10.1590/S0102-311X2008000300004

8. Scherer FC, Martins CR, Pelegrini A, Matheus SC, Petroski EL. Imagem corporal em adolescentes: associação com a maturação sexual e sintomas de transtornos alimentares. J Bras Psiquiatr. 2010; 59(3):198-202. http://dx.doi.org/10.1590/S004720852010000300005

9. Bighetti F, Santos CB, Santos JE, Ribeiro RPP. Tradução e avaliação do Eating Attitudes Test em adolescentes do sexo feminino de Ribeirão Preto, São Paulo. J Bras Psiquiatr. 2004; 53(6):339-46.

10. Fortes LS, Amaral ACS, Almeida SS, Ferreira MEC. Efeitos de variáveis psicológicas, morfológicas e sociodemográficas sobre o comportamento alimentar de adolescentes. Rev Paul Pediatr. 2013; 31(2):182-8. http://dx.doi.org/10.1590/S0103-058 22013000200008

11. Fortes LS, Morgado FFR, Ferreira MEC. Fatores associados ao comportamento alimentar inadequado em adolescentes escolares. Rev Psiquiatr Clin. 2013; 40(2):59-64. http://dx.doi.org/10.1590/ S0101-60832013000200002

12. Martins CR, Pelegrini A, Matheus SC, Petroski EL. Insatisfação com a imagem corporal e a relação com estado nutricional, adiposidade corporal e sintomas de anorexia e bulimia em adolescentes. Rev Psiq. 2010; 32(1):19-23. http://dx.doi.org/10. 1590/S0101-81082010000100004

13. Mousa T, Mashal RH, Al-Domi HA, Jibril MA. Body image dissatisfaction among adolescent schoolgirls in Jordan. Body Image. 2010; 7(1):46-50. http:// dx.doi.org/10.1016/j.bodyim.2009.10.002

14. Amaral ACS, Cordás TA, Conti MA, Ferreira MEC. Equivalência semântica e avaliação da consistência interna da versão em português do Sociocultural Attitudes Towards Appearance Questionnaire-3 (SATAQ-3). Cad Saúde Pública. 2011; 27(8):1487-97. http://dx.doi.org/10.1590/S0102-311X20110008 00004

15. Leal GVS, Philippi ST, Polacow VO, Cordás TA, Alvarenga MS. O que é comportamento de risco para transtornos alimentares em adolescentes? J Bras Psiquiatr. 2013; 62(1):62-75. http://dx.doi.org/ 10.1590/S0047-20852013000100009

16. Flament MF, Hill EM, Buckholz A, Henderson K, Tasca GA. Internalization of the thin and muscular body ideal and disordered eating in adolescence: The mediation effects of body esteem. Body Image. 2012; 9(1):68-75. http://dx.doi.org/10.1016/j. bodyim.2011.07.007

17. Filaire E, Rouveix M, Pannafieux C, Ferrand C. Eating atitudes, perfectionism and body-esteem of elite male judoists and cyclists. J Sports Sci Med. 2007; 6(1):50-7.

18. Rouveix M, Bouget M, Pannafieux C, Champely S, Filaire E. Eating attitudes, body esteem, perfectionism and anxiety of judo athletes and nonathletes. Int J Sports Med. 2007; 28(2):340-5.

19. Sbicigo JB, Bandeira DR, Dell'Aglio DD. Escala de Autoestima de Rosenberg (EAR): validade fatorial e consistência interna. Psico-USF. 2010; 15(3):395-403.

20. Fortes LS, Filgueiras JF, Ferreira MEC. Risk behaviors for eating disorders and depressive symptoms: A study of female adolescents in Juiz de Fora, Minas Gerais State, Brazil. Cad Saúde Pública. 2014; 30(11):2443-50.

21. Fortes LS, Cipriani FM, Almeida SS, Ferreira MEC. Eating disorder symptoms: Association with perfectionism traits in male adolescents. Arch Clin Psychiatry. 2014; 41(5):117-20.

22. Fortes LS, Cipriani FM, Coelho FD, Paes ST, Ferreira MEC. Does self-esteem affect body dissatisfaction levels in female adolescents? Rev Paul Pediatr. 2014; 32(3):236-40.

23. Miranda VPN, Conti MA, Bastos R, Ferreira MEC. Body dissatisfaction in Brazilian adolescents in small towns of Minas Gerais. J Bras Psiquiatr. 2011; 
60(3):190-7. http://dx.doi.org/10.1590/S0047-20 852011000300007

24. Gondoli DM, Corning AF, Salafia EHB, Bucchianeri MM, Fitzsimmons EE. Heterosocial involvement, peer pressure for thinness, and body dissatisfaction among young adolescent girls. Body Image. 2011; 8(2):143-8. http://dx.doi.org/10.1016/j.bodyim.20 10.12.005

25. Caqueo-Urizar A, Ferrer-Garcia M, Toro J, Gutierrez-Maldonado J, Penaloza C, Cuadros-Sosa Y, et al. Associations between sociocultural pressures to be thin, body distress, and eating disorder symptomatology among Chilean adolescent girls. Body Image. 2011; 8(1):78-81. http://dx.doi.org/ 10.1016/j.bodyim.2010.11.004

26. Mirza NM, Davis D, Yanovski JA. Body dissatisfaction, self-esteem and overweight among inner-city Hispanic children and adolescentes. J
Adolescent Health. 2005; 36(3):267-71. http://dx. doi.org/10.1016/j.jadohealth.2004.02.033

27. Johnson C, Crosby R, Engel S, Mitchell J, Powers $P$, Wittrock D, et al. Gender, ethnicity, self-esteem and disordered eating among college athletes. Eat Behav. 2004; 5(1):147-56.

28. Forrester-Knauss C, Perren S, Alsaker FD. Does body mass index in childhood predict restraint eating in early adolescence? Appetite. 2012; 59(3):921-6. http://dx.doi.org/10.1016/j.appet.2012.08.026

29. Fortes LS, Morgado FFR, Almeida SS, Ferreira MEC. Eating behavior and physical activity in adolescents. Rev Nutr. 2013; 26(5):529-37. http://dx.doi.org/ 10.1590/S1415-52732013000500004

Recebido: janeiro 28, 2014 Versão final: fevereiro 20, 2015 Aprovado: março 5, 2015 